\title{
Aroma changes of black tea prepared from methyl jasmonate treated tea plants"
}

\author{
Jiang $\mathrm{SHI}^{1,2}$, Li WANG ${ }^{3}$, Cheng-ying MA ${ }^{1}$, Hai-peng LV $^{1}$, Zong-mao CHEN ${ }^{1}$, Zhi LIN ${ }^{\dagger \neq 1}$ \\ ( ${ }^{I}$ Key Laboratory of Tea Biology and Resource Utilization of Ministry of Agriculture, Tea Research Institute, \\ Chinese Academy of Agricultural Sciences, Hangzhou 310008, China) \\ $\left({ }^{2}\right.$ Graduate School of Chinese Academy of Agricultural Sciences, Beijing 100081, China) \\ ( ${ }^{3}$ Lin'an Agriculture Bureau, Lin'an 311300, China) \\ †E-mail: linz@tricaas.com
}

Received Sept. 3, 2013; Revision accepted Dec. 15, 2013; Crosschecked Mar. 18, 2014

\begin{abstract}
Methyl jasmonate (MeJA) was widely applied in promoting food quality. Aroma is one of the key indicators in judging the quality of tea. This study examined the effect of exogenous MeJA treatment on tea aroma. The aroma components in black tea prepared from MeJA-treated fresh tea leaves were extracted using headspace solid-phase microextraction (HS-SPME) and were analyzed using gas chromatography-mass spectrometry (GC-MS) and GC-olfactometry (GC-O). Forty-five volatile compounds were identified. The results revealed that the MeJA-treated black tea had higher levels of terpene alcohols and hexenyl esters than the untreated tea. Moreover, several newly components, including copaene, cubenol, and indole, were induced by the MeJA treatment. The activities of polyphenol oxidase and $\beta$-glucosidase in fresh tea leaves changed after the MeJA treatment. Quantitative real-time polymerase chain reaction (qRT-PCR) analysis indicated that the gene expression levels of polyphenol oxidase and $\beta$-primeverosidase were upregulated by two and three folds, respectively, by the MeJA treatment $(P<0.01)$; however, the gene expression of $\beta$-glucosidase was downregulated to a half level. In general, the aroma quality of the MeJAtreated black tea was clearly improved.
\end{abstract}

Key words: Aroma, Black tea, Methyl jasmonate (MeJA), Headspace solid-phase microextraction (HS-SPME), Gas chromatography-mass spectrometry (GC-MS), Gas chromatography-olfactometry (GC-O), Gene expression doi: $10.1631 /$ jzus.B1300238

Document code: A

CLC number: Q58

\section{Introduction}

Tea (Camellia sinensis) is the most widely consumed non-alcoholic beverage in the world, with several different types available, such as green tea, oolong tea, and black tea. Black tea is a kind of fully fermented tea and is manufactured in four distinct stages, including withering, rolling, fermentation, and drying (Tomlins and Mashingaidze, 1997). The

\footnotetext{
${ }^{\ddagger}$ Corresponding author

* Project supported by the National Natural Science Foundation of China (No. 31270734), the Construction Project of Modern Agricultural Technology System (No. CARS-23), and the Zhejiang Provincial Science and Technology Plan Project (No. 2007C12G3020014), China (C) Zhejiang University and Springer-Verlag Berlin Heidelberg 2014
}

worldwide popularity of black tea has been attributed to its sensory quality, with particular emphasis on its unique aroma and characteristic flavor. Hence, the specific aroma profile is a key factor in determining the quality grade of tea.

Aroma compounds are key contributors to tea flavor perception. A variety of different volatile organic components (VOCs) are presented in tea although in minute quantities (i.e., $0.01 \%$ of the total dry weight). VOCs have a significant impact on tea flavor because of their low threshold values and resulting high odor units (Rawat et al., 2007). Generally, aroma compounds are classified into two groups: Group I is defined as non-terpenoid species, for instance, hexanal, trans-2-hexenal, 2-hexanol, and 
cis-3-hexenol; Group II is defined as terpenoid species, such as linalool, linalool oxides, benzaldehyde, phenylacetaldehyde, nerol, geraniol, $\alpha$-ionone, benzyl alcohol, phenyl ethanol, and $\beta$-ionone (Muthumani et al., 2013). The ratio of terpenoid species to nonterpenoid species has been used to classify black teas for their flavor quality. In particular, linalool and geraniol, which belong to the terpene alcohol family, always impart a sweet, flowery aroma on black tea (Sanderson et al., 1973; Vitzthum et al., 1975; Kawakami et al., 1995).

It is well known that the aroma composition of tea varies with various parameters, such as plant variety, plucking season, soil, climate, pre- and post-harvest treatments (Qin et al., 2013). Moreover, the flavor of prepared tea could also be affected by insect infestation, stresses, and mechanical injury to tea plants (Bailey et al., 2005; Bhattacharyya et al., 2007; Cho et al., 2007). Methyl jasmonate (MeJA), a well-characterized fatty acid-derived cyclopentanone signal, can induce a series of changes in secondary metabolites in a wide range of plants (Alvarez et al., 2009; Kuźma et al., 2009; Qiu et al., 2009; Chen et al., 2011). MeJA plays an important role in promoting the quality of agricultural products, especially in improving the aroma qualities of certain fruits and vegetables. Kuroyanagi et al. (1998) isolated seven sesquiterpenoid phytoalexins from the culture medium of hairy roots of Hyoscyamus albus following its treatment with MeJA. Loivamäki et al. (2004) also reported that jasmonate induction led to an increase in tobacco alkaloids, phenolics, and diterpene glycosides in Nicotiana attenuate. Moreover, the impacts of MeJA on the biosynthesis of volatile compounds in climacteric and non-climacteric fruits, such as apples and strawberries, respectively, have also been reported (Ayala-Zavala et al., 2005; Kim et al., 2006; de la Peña Moreno et al., 2010a; 2010b; Belhadj et al., 2008; Gohain et al., 2012). However, investigations toward the effects of exogenous MeJA on tea aroma have been scarcely reported.

In this study, the aroma components of black tea prepared from MeJA-treated tea plants were extracted using headspace solid-phase microextraction (HSSPME) and analyzed using gas chromatography-mass spectrometry (GC-MS) (Lin et al., 2013). Moreover, the characteristic aroma components of the prepared tea were further identified using GC-olfactometry
(GC-O) (Lv et al., 2012). To determine the mechanism of action, we studied the enzyme activities and gene expressions of several aroma-related enzymes, such as polyphenol oxidase (PPO), $\beta$-glucosidase, and $\beta$-primeverosidase. After clarifying the variation of tea aroma induced by exogenous MeJA, this study could provide a theoretical basis and technical support for using an exogenous inducer to enhance aroma quality and developing a new flavored black tea.

\section{Materials and methods}

\subsection{Reagents}

MeJA ( $>98 \%$ ) was purchased from Sigma-Aldrich Co. (USA). Pure water was obtained from a Milli-Q purification system (Millipore, USA). A $65-\mu \mathrm{m}$ SPME head [(polydimethylsiloxane/divinylbenzene (PDMS/DVB)] fiber was obtained from Supelco (Bellefonte, USA). The RNAplant plus kit, FastQuant RT kit (with gDNase), and SuperReal PreMix Plus (SYBR Green) were purchased from TIANGEN Biotech Co., Ltd., China.

\subsection{Tea samples and preparations}

Longjing 43, a cultivar of the tea plant (Camellia sinensis), was grown in the garden of the Tea Research Institute, Chinese Academy of Agricultural Sciences, Hangzhou, China. Samples were treated and prepared in spring. All the experiments were carried out in triplicate and separately in March, April, and May.

Preparation of MeJA-treated black tea: 1000 individual tea plants were homogeneously sprayed with $4 \mathrm{~L} \quad 0.25 \%(\mathrm{w} / \mathrm{v})$ solution of MeJA dissolved in ethanol. The fresh tea leaves were then allowed to stand for $24 \mathrm{~h}$ before being plucked (one bud with the second and third leaves). The leaves (2000 g) were then processed as follows: withered for $45 \mathrm{~h}$ at room temperature, rolled for $40 \mathrm{~min}$ in a proper rolling machine, and then subjected to a fermentation process before being dried at $120{ }^{\circ} \mathrm{C}$ to provide the finished black tea $(500 \mathrm{~g})$.

Untreated black tea was used as the control. It was prepared using water instead of MeJA for spraying the tea plants, and other processings were the same as the above described MeJA-treated black tea. 


\subsection{HS-SPME procedure}

Extraction of the volatile compounds was performed by an HS-SPME method using a PDMS/DVB fiber. Before analysis, the fiber was preconditioned for $5 \mathrm{~min}$ in the injector of the GC as indicated by instructions of the manufacturer.

For each sample, $10.0 \mathrm{~g}$ of tea, previously homogenized, was weighed in a $150-\mathrm{ml}$ vial and infused with $30 \mathrm{ml}$ of boiling water. The vial was then sealed with tetrafluoroethylene and immediately heated to and held at $60{ }^{\circ} \mathrm{C}$. Prior to extraction, an incubation time of $5 \mathrm{~min}$ was applied to equilibrate the fiber. The SPME fiber was then successively exposed to the headspace for $60 \mathrm{~min}$ while maintaining the sample temperature at $60{ }^{\circ} \mathrm{C}$. Following the sampling process, the SPME fiber was injected into the GC injector and left for $3.5 \mathrm{~min}$ to allow for the thermal desorption of the analytes.

Based on our trials, the best extraction conditions were defined as follows: a $65-\mu \mathrm{m}$ SPME head (PDMS/DVB) fiber with extraction time of $60 \mathrm{~min}$, an extraction temperature of $60{ }^{\circ} \mathrm{C}$ in a water bath, and a 1:3 brewing ratio of tea to water.

\subsection{GC-MS analyses}

GC-MS analyses were conducted on an Agilent6890 GC directly coupled to an Agilent HP 5973 MSD ion trap mass spectrometer (Agilent, USA). An injector temperature of $250{ }^{\circ} \mathrm{C}$ was used in the splitless injection mode. An HP-5MS column (30 m× $0.25 \mathrm{~mm}$ i.d., $0.25 \mu \mathrm{m}$ film thickness) was used for the analyses. The oven temperature was programmed as follows: the initial temperature was held at $50{ }^{\circ} \mathrm{C}$ for $5 \mathrm{~min}$, and the column was then heated at $3{ }^{\circ} \mathrm{C} / \mathrm{min}$ to $210{ }^{\circ} \mathrm{C}$ and subsequently held for $3 \mathrm{~min}$ before being heated at $15^{\circ} \mathrm{C} / \mathrm{min}$ to $230{ }^{\circ} \mathrm{C}$ and finally to $250{ }^{\circ} \mathrm{C}$. Helium (purity $>99.999 \%$ ) was used as the carrier gas at a constant flow rate of $1 \mathrm{ml} / \mathrm{min}$.

The mass spectra were collected in the electron impact ionization mode, with an electron energy of $70 \mathrm{eV}$, interface temperature of $280{ }^{\circ} \mathrm{C}$, ion source temperature of $230{ }^{\circ} \mathrm{C}$, quadrupole temperature of $150{ }^{\circ} \mathrm{C}$, mass scan range of 35-400 atomic mass units, and an emission current of $34.6 \mu \mathrm{A}$. Each compound was identified using the National Institute of Standards and Technology (NIST) 98 library based on their linear retention indices (LRI) and authentic standards. The relative proportions of the constituents were obtained by flame ionization detector (FID) peak area normalization. Quantitative results were obtained using the following equation: relative content $(\%)=$ single constituent area $\times 100 \% /$ total area.

\subsection{Sensory evaluation of tea aroma}

Small portions $(3.0 \mathrm{~g})$ of the MeJA-treated and the untreated black tea were accurately weighed and placed in $150 \mathrm{ml}$ of boiling water to brew the tea for a period of $5 \mathrm{~min}$. Five professional tea tasters were then invited to perform a sensory evaluation and objective assessment of the aroma of tea.

\subsection{Determination of activities and gene expres- sions of enzymes in fresh tea leaves}

\subsubsection{Activities of PPO and $\beta$-glucosidase}

PPO and $\beta$-glucosidase were extracted and their acitivities were determined according to Wang et al. (2001) and Wakuta et al. (2010). However, because of a lack of $\beta$-primeveroside as the substrate, the activity of $\beta$-primeverosidase was not determined.

2.6.2 Gene expressions of PPO, $\beta$-glucosidase, and $\beta$-primeverosidase

Freshly prepared tea leaves $(100 \mathrm{mg})$ were immediately ground in liquid nitrogen, and a portion of this sample powder was used for RNA extraction with the RNAprep Pure Plant Kit (TIANGEN Biotech Co., Ltd., Beijing, China). Complementary DNA (cDNA) was synthesized using the FastQuant RT Kit (TIANGEN Biotech Co., Ltd., Beijing, China). Quantitative real-time polymerase chain reaction (qRT-PCR) was carried out using SuperReal PreMix Plus (SYBR Green) with the first-strand cDNA as a template on the ABI 7500 Real-Time PCR System (Applied Biosystems). The relative gene expressions of PPO, $\beta$ glucosidase, and $\beta$-primeverosidase were determined.

\subsection{Statistical analysis}

Data were statistically analyzed by SPSS statistical package (Version 16.0 for Windows). One-way analysis of variance (ANOVA) was used to test the hypothesis and the significant differences were considered when $P<0.05$. Data were expressed as mean \pm standard deviation (SD). 


\section{Results}

\subsection{Identification and quantification of volatile components in MeJA-treated and untreated teas}

Table 1 shows the differences in the relative content of each volatile compound extracted from the MeJA-treated and untreated black teas. A total of 42 major volatile compounds were identified using GC-MS and were grouped into different classes, including 9 terpene alcohols, 7 terpenes, 3 newly induced components, 7 hexenyl esters, and 16 other components. As shown in Fig. 1, significant differences of most volatile compounds were observed between the MeJA-treated and untreated black teas. Increases in the production of these compounds could eventually enhance the sensory quality of freshly prepared black tea.

The flavor index was calculated according to Yamanishi et al. (1968a; 1968b) as the ratio of the sum of relative distributions of Group II compounds to the sum of relative distributions of Group I compounds. The flavor index of the MeJA-treated black tea increased to almost 2.65; however, that of the untreated black tea was only 2.15 . Based on this consideration, the sensory quality of MeJA-treated black tea was clearly improved.

\subsection{Differences among terpene alcohols}

Nine different terpene alcohols were found in the prepared black tea (Table 1). Geraniol was found to be the most abundant of these nine alcohols, followed by $\beta$-linalool, the levels of which were both increased due to the MeJA treatment. The total relative content of $\beta$-linalool and its oxides, together with geraniol, was $63.91 \%$ in the untreated black tea, whereas the corresponding value in the MeJA-treated black tea was $68.20 \%$. The total content of terpene alcohols in the MeJA-treated black tea was significantly higher than that of the control $(P<0.05$; Fig. 1). It was reported that $\beta$-linalool and geraniol play important roles in the formation of black tea aroma (Schuh and Schieberle, 2006). $\beta$-Linalool and its corresponding oxides contribute vitally to the formation of the sweet aroma of black tea, whereas geraniol has an effect on the formation of the floral aroma of black tea. In this study, MeJA could induce the production of terpene alcohols, especially $\beta$-linalool and geraniol, which contributed significantly to the total percentage of volatile compounds, resulting in an improved aroma quality and possibly accounting for the sweet and floral aroma to a great degree.

\subsection{Differences among hexenyl esters}

Hexenyl esters are the second important class of compounds presented in the black teas (Table 1). The relative contents of these seven hexenyl esters also changed following the MeJA treatment. Notably, the relative content of cis-hexanoic acid 3-hexenyl ester increased from $2.6 \%$ to $3.2 \%$ due to the MeJA treatment. Furthermore, a pair of diastereoisomers, cis-hexanoic acid 3-hexenyl ester and 3-hexenylhexanoic acid ester, were presented in the overall volatile composition. The ratio of cis-hexanoic acid 3-hexenyl ester to 3-hexenyl-hexanoic acid ester was about 5:1 in the untreated black tea, whereas this ratio dropped to about 3:1 in the MeJA-treated black tea. Moreover, our previous research indicated that cishexanoic acid 3-hexenyl ester could be responsible for the floral aroma of freshly prepared black tea (data unpublished). Appropriate aroma and flavor components always yield higher quality of tea. In a similar research conducted on the MeJA-treated strawberries, MeJA could affect the enantiomeric distribution of ethyl 2-methylbutanoate, which also exerted a significant impact on aroma formation of strawberries (Blanch et al., 2011). Likewise, it is speculated that MeJA treatment of black tea in the current study affected the enantiomeric distribution of hexanoic acid3-hexene ester. MeJA treatment could play a significant role in affecting the aromas of different black teas, depending on different ratios of the isomers.

\subsection{Differences among other volatiles}

It was interesting to note that MeJA could induce several new secondary metabolite components, which was in agreement with previous studies (RodriguezSaona et al., 2001; Martin et al., 2003; Degenhardt and Lincoln, 2006). Three new components, copaene, cubenol, and indole, were induced in the MeJAtreated tea but not in the untreated tea; their relative contents were $0.21 \%, 0.28 \%$, and $0.15 \%$, respectively. However, these components were not detected by GC-MS in the untreated tea. Based on our previous GC-O results, copaene could provide a floral aroma and cubenol could provide a clean and fresh aroma, whereas indole provides a pungent aroma. 
Table 1 GC-MS identification of black tea prepared from MeJA-treated and untreated tea plants

\begin{tabular}{|c|c|c|c|c|c|}
\hline \multirow{2}{*}{ Aroma compound } & \multirow{2}{*}{ LRI } & \multicolumn{2}{|c|}{ Relative content (\%) } & \multirow{2}{*}{$P$} & \multirow{2}{*}{$\begin{array}{c}\text { Identification } \\
\text { method }\end{array}$} \\
\hline & & Untreated & MeJA-treated & & \\
\hline \multicolumn{6}{|l|}{ Terpene alcohol } \\
\hline$\beta$-Linalool & 1080 & $13.73 \pm 0.50$ & $15.15 \pm 0.45$ & $<0.05$ & LRI, MS, Std \\
\hline cis-Linalool oxide (furanoid) & 1087 & $4.30 \pm 0.78$ & $4.95 \pm 0.27$ & & LRI, MS, Std \\
\hline trans-Linalool oxide (furanoid) & 1073 & $9.67 \pm 0.34$ & $10.97 \pm 0.28$ & $<0.05$ & LRI, MS, Std \\
\hline cis-Linalool oxide (pyranoid) & 1174 & $0.91 \pm 0.10$ & $0.70 \pm 0.06$ & $<0.05$ & LRI, MS tent. \\
\hline trans-Linalool oxide (pyranoid) & 1167 & $1.80 \pm 0.12$ & $1.80 \pm 0.10$ & & LRI, MS tent. \\
\hline 3-Hexen-1-ol & 858 & $1.41 \pm 0.06$ & $0.46 \pm 0.13$ & $<0.01$ & LRI, MS, Std \\
\hline Phenylethyl alcohol & 1114 & $2.81 \pm 0.21$ & $2.34 \pm 0.49$ & & LRI, MS, Std \\
\hline cis-Geraniol & 1229 & $27.7 \pm 0.82$ & $28.87 \pm 0.07$ & & LRI, MS, Std \\
\hline cis-Nerolidol & 1573 & $1.58 \pm 0.10$ & $2.23 \pm 0.12$ & $<0.01$ & LRI, MS, Std \\
\hline \multicolumn{6}{|l|}{ Hexenyl ester } \\
\hline cis-Butanoic acid, 3-hexenyl ester & 1212 & $0.46 \pm 0.08$ & $0.53 \pm 0.09$ & & LRI, MS, Std \\
\hline cis-3-Hexenyl iso-valerate & 1238 & $2.64 \pm 0.07$ & $2.62 \pm 0.36$ & $<0.01$ & LRI, MS, Std \\
\hline$n$-Valeric acid cis-3-hexenyl ester & 1230 & $0.56 \pm 0.08$ & $0.63 \pm 0.05$ & & LRI, MS, Std \\
\hline cis-Hexanoic acid, 3-hexenyl ester & 1369 & $2.60 \pm 0.22$ & $3.13 \pm 0.04$ & $<0.05$ & LRI, MS, Std \\
\hline 3-Hexenyl-hexanoic acid ester & 1371 & $0.49 \pm 0.20$ & $1.15 \pm 0.10$ & $<0.05$ & LRI, MS, Std \\
\hline trans-2-Hexenyl caproate & 1391 & $0.31 \pm 0.10$ & $0.28 \pm 0.14$ & & LRI, MS, Std \\
\hline cis-3-Hexenyl benzoate & 1615 & $0.37 \pm 0.17$ & $0.53 \pm 0.09$ & & LRI, MS, Std \\
\hline \multicolumn{6}{|l|}{ New component } \\
\hline$\alpha$-Copaene & 1378 & & $0.16 \pm 0.06$ & $<0.01$ & LRI, MS, Std \\
\hline Cubenol & 1650 & & $0.21 \pm 0.10$ & $<0.01$ & LRI, MS, Std \\
\hline Indole & 1294 & & $0.20 \pm 0.08$ & $<0.01$ & LRI, MS, Std \\
\hline \multicolumn{6}{|l|}{ Terpene } \\
\hline$\beta$-Myrcene & 981 & $2.67 \pm 0.37$ & $2.82 \pm 0.14$ & & LRI, MS, Std \\
\hline$\alpha$-Cubebene & 1354 & $0.35 \pm 0.11$ & $0.39 \pm 0.05$ & & LRI, MS, Std \\
\hline$\beta$-Cubebene & 1388 & $0.36 \pm 0.13$ & $0.37 \pm 0.05$ & & LRI, MS, Std \\
\hline$\alpha$-Cedrene & 1432 & $0.45 \pm 0.07$ & $0.41 \pm 0.04$ & & LRI, MS, Std \\
\hline Caryophyllene oxide & 1589 & $0.14 \pm 0.13$ & $0.41 \pm 0.12$ & $<0.01$ & LRI, MS, Std \\
\hline$\alpha$-Farnesene & 1505 & $0.14 \pm 0.14$ & $0.30 \pm 0.11$ & $<0.01$ & LRI, MS, Std \\
\hline$\delta$-Cadinene & 1528 & $0.26 \pm 0.62$ & $0.48 \pm 0.15$ & $<0.05$ & LRI, MS, Std \\
\hline \multicolumn{6}{|l|}{ Other component } \\
\hline Salicylic acid, methyl ester & 1185 & $4.77 \pm 0.64$ & $5.53 \pm 0.43$ & & LRI, MS, Std \\
\hline Benzaldehyde & 960 & $3.30 \pm 0.13$ & $1.63 \pm 0.26$ & $<0.01$ & LRI, MS, Std \\
\hline Benzene acetaldehyde & 1049 & $3.67 \pm 0.17$ & $2.81 \pm 0.16$ & $<0.01$ & LRI, MS, Std \\
\hline Decanal & 1200 & $1.45 \pm 0.10$ & $0.34 \pm 0.08$ & $<0.01$ & LRI, MS, Std \\
\hline$\beta$-Cyclocitral & 1208 & $0.57 \pm 0.20$ & $0.22 \pm 0.09$ & & LRI, MS, Std \\
\hline cis-Jasmone & 1406 & $0.67 \pm 0.10$ & $0.85 \pm 0.08$ & & LRI, MS, Std \\
\hline Geranyl acetone & 1456 & $3.33 \pm 0.38$ & $1.59 \pm 0.41$ & $<0.05$ & LRI, MS, Std \\
\hline$\beta$-Ionone & 1493 & $1.10 \pm 0.48$ & $0.42 \pm 0.14$ & & LRI, MS, Std \\
\hline 2,6-Di-tert-butyl-4-methyl phenol & 1512 & $0.57 \pm 0.10$ & $0.61 \pm 0.13$ & & LRI, MS, Std \\
\hline 2-Methylnaphthalene & 1884 & $0.56 \pm 0.16$ & $0.52 \pm 0.12$ & & LRI, MS, Std \\
\hline Tetradecane & 239 & $1.54 \pm 0.25$ & $1.07 \pm 0.11$ & & LRI, MS, Std \\
\hline 2,6,10,14-Tetramethyl-heptadecanoic & 1872 & $0.60 \pm 0.12$ & $0.56 \pm 0.05$ & & LRI, MS, Std \\
\hline$n$-Pentadecane & 253 & $0.75 \pm 0.10$ & $0.65 \pm 0.06$ & & LRI, MS, Std \\
\hline 1-Hexadecene & 1587 & $0.55 \pm 0.10$ & $0.35 \pm 0.12$ & & LRI, MS, Std \\
\hline$n$-Hexadecane & 272 & $0.52 \pm 0.10$ & $0.59 \pm 0.10$ & & LRI, MS, Std \\
\hline 2,6,10,14-Tetramethyl-pentadecane & 1707 & $0.33 \pm 0.17$ & $0.26 \pm 0.10$ & & LRI, MS, Std \\
\hline
\end{tabular}

Data are expressed as mean \pm SD of three replicates. LRI: linear retention index (calculated on HP-5MS column). MS tent.: tentatively identified by MS; Std: chemical standard. When only MS or LRI is available for the identification of a compound, it must be considered as an attempt of identification 


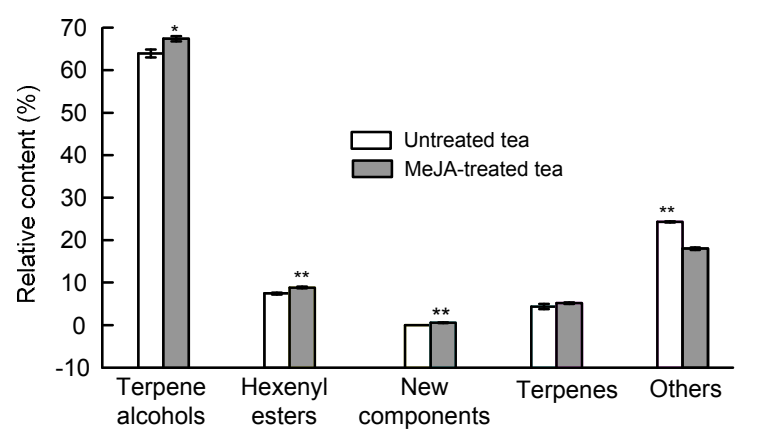

Fig. 1 Relative contents of different classifications of volatile components presented in MeJA-treated and untreated black teas

Data are expressed as mean $\pm \operatorname{SD}(n=3) .{ }^{*} P<0.05 ;{ }^{* *} P<0.01$

The terpenes in the MeJA-treated black tea, including $\beta$-myrcene, $\alpha$-cubebene, $\beta$-cubebene, $\alpha$-cedrene, caryophyllene oxide, $\alpha$-farnesene, and $\delta$-cadinene, also differed considerably from those of the untreated tea. The relative contents of caryophyllene oxide, $\alpha$-farnesene, and $\delta$-cadinene in the MeJA-treated black tea were significantly higher (Table 1; $P<0.05$ ).

As shown in Fig. 1, each of these compound classes underwent changes by the MeJA treatment, clearly indicating that MeJA had a significant impact on the formation of the overall tea aroma.

\subsection{Sensory evaluations of aromas of the MeJA- treated and untreated teas}

Based on the results from the five tea tasters, it became clear that the sensory quality of the aroma of black tea prepared with the MeJA-treated fresh leaves was enhanced relative to the control. As shown in Table 2, the MeJA-treated black tea was given a score of 91.7 in the sensory evaluation, whereas the untreated black tea was given a score of 88.7. Furthermore, the types of aromas were quite different: the MeJA-treated black tea possessed a honey aroma, whereas the control had a sweet aroma which was similar to normal black tea. These results therefore demonstrated that MeJA treatment induced differences in the aroma of the tea as well as enhancing the sensory quality of the aroma.

\subsection{Activities of PPO and $\beta$-glucosidase}

As shown in Fig. 2, MeJA treatment significantly improved the acitivty of PPO $(P<0.01)$, while decreased the activity of $\beta$-glucosidase $(P<0.01)$.
3.7 Gene expressions of PPO, $\beta$-glucosidase, and $\beta$-primeverosidase

PPO, $\beta$-glucosidase, and $\beta$-primeverosidase were the three most important enzymes in the release of free aroma from tea leaves. The results of qRT-PCR showed that the gene expression levels of PPO and $\beta$-primeverosidase were significantly upregulated in the MeJA-treated tea leaves $(P<0.01)$. However, the gene expression of $\beta$-glucosidase was downregulated by the MeJA treatment (Fig. 3). These results were in accordance with the activities of these enzymes.

Table 2 Sensory evaluations of MeJA-treated and untreated black teas

\begin{tabular}{lll}
\hline \multicolumn{1}{c}{ Tea } & \multicolumn{1}{c}{ Sensory remark } & \multicolumn{1}{c}{ Score } \\
\hline Untreated & Sweet aroma & $88.7 \pm 0.5$ \\
MeJA-treated & Honey-sweet aroma & $91.7 \pm 1.5^{*}$ \\
\hline
\end{tabular}

Data are expressed as mean $\pm \mathrm{SD}$ of three replicates. ${ }^{*} P<0.05$

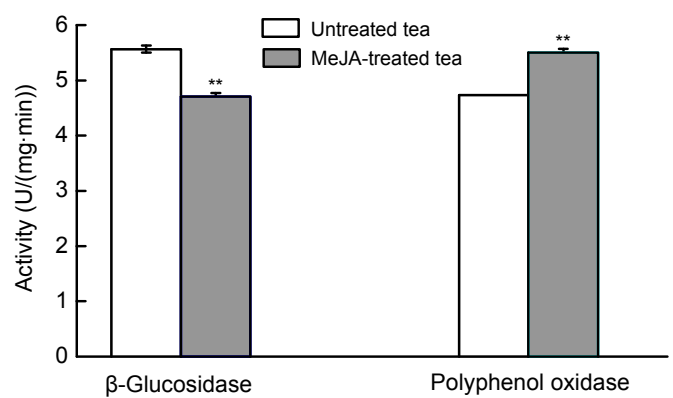

Fig. 2 Effects of exogenous methyl jasmonate on the activities of $\boldsymbol{\beta}$-glucosidase and polyphenol oxidase Data are expressed as mean $\pm \operatorname{SD}(n=3) .{ }^{* *} P<0.01$

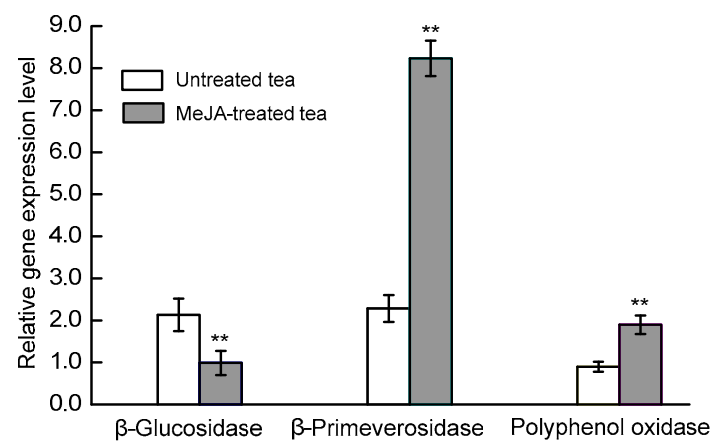

Fig. 3 Effects of exogenous MeJA on gene expressions of $\beta$-glucosidase, $\beta$-primeverosidase, and ployphenol oxidase in tea leaves

Data are expressed as mean $\pm \mathrm{SD}(n=3) .{ }^{* *} P<0.01$ 


\section{Discussion}

Some other studies proved the theory that tea aroma arose partly from the fresh leaves and partly from the manufacturing process. Although special manufacturing processes give black tea a unique floral, fruity, and honey-sweet aroma, to date, little attention has been given to exogenous effects on fresh tea leaves which affect the final black tea aroma. This study is the first to demonstrate that the aroma of black tea prepared from MeJA-treated tea plants is greatly improved.

The basic structure of MeJA is effectively a type of cyclopentane ketone which is a naturally volatile compound itself. MeJA could be hydrolyzed by an esterase enzyme in plant cells into jasmonate, which then works in the jasmonate pathway (Lyons et al., 2013). Importantly, this pathway could then exert a significant influence on certain aroma-related enzymes, especially $\beta$-glucosidase, $\beta$-primeverosidase, and PPO (Wakuta et al., 2010; Yang et al., 2013).

In this work, MeJA treatment could significantly enhance the gene expressions of $\beta$-primeverosidase and PPO, although suppressed the gene expression of $\beta$-glucosidase in fresh tea leaves. $\beta$-Primeverosidase and $\beta$-glucosidase are both crucial catalysts for the hydrolysis reactions of the glycoside precursors of the corresponding free aroma compounds (Wang et al., 2001; Sarry and Günata, 2004). Tea volatile components exist, to a significant degree, as glycosidebound aromas (glycoside precursors) in fresh tea leaves. Only after coming into contact with hydrolases, mainly $\beta$-glucosidase and $\beta$-primeverosidase, can these conjugated aroma compounds be released as the free type during the hydrolysis reaction (Ogawa et al., 1997; Ijima et al., 1998). Among the glycosidic aroma precursors, $\beta$-primeveroside is usually considered the prevalent glucoside. Modulation of gene expression may result in improving enzyme activity. It can be deduced that the improved activity of $\beta$-primeverosidase is mainly responsible for the release of terpene alcohols, such as $\beta$-linalool, linalool oxides, and geraniol, which account for the greatest proportion of tea aroma. That is, MeJA could potentially induce the enhancement of $\beta$-primeverosidase activity, which ultimately results in releasing terpene alcohol aromas. As for PPO, the mechanism seems much more complicated. PPO could oxidize catechins, the predominant chemical components in tea, into the corresponding oxidized catechin species. These oxidized catechins could then undergo condensation reactions with other types of catechins to generate theaflavins (TF) or even thearubigins (TR). Furthermore, the oxidized catechins could affect $\beta$ carotene, resulting in its degradation to $\beta$-ionone, which has a woody and floral aroma. In addition, the oxidized catechins could react with other carotene species to form ketone aroma compounds (e.g., $\beta$-ionone or $\alpha$-ionone). The compounds produced from carotenoids also have a remarkable effect on the aroma of tea (Ravichandran, 2002). Hence, we deduced that the MeJA treatment on fresh tea leaves has a positive effect on several crucial aroma-related enzymes that ultimately result in the promotion of black tea aroma quality.

In the present study, MeJA has been used as an exogenous inducer to improve the gene expressions and the activities of PPO and $\beta$-primeverosidase in tea leaves. The activity promotions of these enzymes eventually had an effect on the sensory quality of the tea aroma. The characteristic aromas of the black tea prepared from MeJA-treated fresh tea leaves were identified using an HS-SPME method combined with GC-MS. The results provide good qualitative and quantitative evidences that we have succeeded in promoting the levels of all selected volatile compounds of black tea by the MeJA treatment. To the best of our knowledge, this is the first time to report the use of MeJA for improving the sensory quality of tea aroma. However, further investigation aimed at determining an accurate regulatory mechanism for MeJA treatment on tea aroma should be conducted for a better understanding of the overall process.

\section{Conclusions}

Significant differences in aroma components were found in black tea prepared from MeJA-treated tea plants. In general, the sensory quality of tea aroma was clearly improved. MeJA treatment could affect the expression of certain genes that modulate the relevant enzyme activity, resulting in increased free terpene alcohol aromas.

\section{Compliance with ethics guidelines}

Jiang SHI, Li WANG, Cheng-ying MA, Hai-peng LV, Zong-mao CHEN, and Zhi LIN declare that they have no conflict of interest. 
This article does not contain any studies with human or animal subjects performed by any of the authors.

\section{References}

Alvarez, S., Zhu, M., Chen, S., 2009. Proteomics of Arabidopsis redox proteins in response to methyl jasmonate. $J$. Proteom., 73(1):30-40. [doi:10.1016/j.jprot.2009.07.005] Ayala-Zavala, J.F., Wang, S.Y., Wang, C.Y., et al., 2005. Methyl jasmonate in conjunction with ethanol treatment increases antioxidant capacity, volatile compounds and postharvest life of strawberry fruit. Eur. Food Res. Technol., 221(6):731-738. [doi:10.1007/s00217-005-0069-z]

Bailey, B.A., Strem, M.D., Bae, H., et al., 2005. Gene expression in leaves of Theobroma cacao in response to mechanical wounding, ethylene, and/or methyl jasmonate. Plant Sci., 168(5):1247-1258. [doi:10.1016/j.plantsci. 2005.01.002]

Belhadj, A., Telef, N., Saigne, C., et al., 2008. Effect of methyl jasmonate in combination with carbohydrates on gene expression of PR proteins, stilbene and anthocyanin accumulation in grapevine cell cultures. Plant Physiol. Biochem., 46(4):493-499. [doi:10.1016/j.plaphy.2007.12. 001]

Bhattacharyya, N., Seth, S., Tudu, B., et al., 2007. Monitoring of black tea fermentation process using electronic nose. $J$. Food Eng., 80(4):1146-1156. [doi:10.1016/j.jfoodeng. 2006.09.006]

Blanch, G.P., Flores, G., Ruiz del Castillo, M.L., 2011. Influence of methyl jasmonate in conjunction with ethanol on the formation of volatile compounds in berries belonging to the Rosaceae. Postharv. Biol. Technol., 62(2): 168-178. [doi:10.1016/j.postharvbio.2011.05.003]

Chen, Y., Pang, Q., Dai, S., et al., 2011. Proteomic identification of differentially expressed proteins in Arabidopsis in response to methyl jasmonate. J. Plant Physiol., 168(10): 995-1008. [doi:10.1016/j.jplph.2011.01.018]

Cho, J.Y., Mizutani, M., Shimizu, B.I., et al., 2007. Chemical profiling and gene expression profiling during the manufacturing process of Taiwan oolong tea "Oriental Beauty”. Biosci. Biotechnol. Biochem., 71(6):1476-1486. [doi:10.1271/bbb.60708]

Degenhardt, D.C., Lincoln, D.E., 2006. Volatile emissions from an odorous plant in response to herbivory and methyl jasmonate exposure. J. Chem. Ecol., 32(4):725-743. [doi:10.1007/s10886-006-9030-2]

de la Peña Moreno, F., Blanch, G.P., Flores, G., et al., 2010a. Development of a method based on on-line reversed phase liquid chromatography and gas chromatography coupled by means of an adsorption-desorption interface for the analysis of selected chiral volatile compounds in methyl jasmonate treated strawberries. J. Chromatogr. A, 1217(7):1083-1088. [doi:10.1016/j.chroma.2009.10.037]

de la Peña Moreno, F., Blanch, G.P., Flores, G., et al., 2010b. Impact of postharvest methyl jasmonate treatment on the volatile composition and flavonol content of strawberries. J. Sci. Food Agric., 90(6):989-994. [doi:10.1002/jsfa.
3908]

Gohain, B., Borchetia, S., Bhorali, P., et al., 2012. Understanding Darjeeling tea flavour on a molecular basis. Plant Mol. Biol., 78(6):577-597. [doi:10.1007/s11103012-9887-0]

Ijima, Y., Ogawa, K., Watanabe, N., et al., 1998. Characterization of $\beta$-primeverosidase, being concerned with alcoholic aroma formation in tea leaves to be processed into black tea, and preliminary observations on its substrate specificity. J. Agric. Food Chem., 46(5):1712-1718. [doi:10.1021/jf970576g]

Kawakami, M., Ganguly, S.N., Banerjee, J., et al., 1995. Aroma composition of oolong tea and black tea by brewed extraction method and characterizing compounds of Darjeeling tea aroma. J. Agric. Food Chem., 43(1): 200-207. [doi:10.1021/jf00049a037]

Kim, H.J., Chen, F., Wang, X., et al., 2006. Effect of methyl jasmonate on secondary metabolites of sweet basil (Ocimum basilicum L.). J. Agric. Food Chem., 54(6): 2327-2332. [doi:10.1021/jf051979g]

Kuroyanagi, M., Arakawa, T., Mikami, Y., et al., 1998. Phytoalexins from hairy roots of Hyoscyamus albus treated with methyl jasmonate. J. Nat. Prod., 61(12):1516-1519. [doi:10.1021/np980214i]

Kuźma, Ł., Bruchajzer, E., Wysokińska, H., 2009. Methyl jasmonate effect on diterpenoid accumulation in Salvia sclarea hairy root culture in shake flasks and sprinkle bioreactor. Enzyme Microb. Technol., 44(6-7):406-410. [doi:10.1016/j.enzmictec.2009.01.005]

Lin, J., Zhang, P., Pan, Z., et al., 2013. Discrimination of oolong tea (Camellia sinensis) varieties based on feature extraction and selection from aromatic profiles analyzed by HS-SPME/GC-MS. Food Chem., 141(1):259-265. [doi:10.1016/j.foodchem.2013.02.128]

Loivamäki, M., Holopainen, J.K., Nerg, A.M., 2004. Chemical changes induced by methyl jasmonate in oilseed rape grown in the laboratory and in the field. J. Agric. Food Chem., 52(25):7607-7613. [doi:10.1021/jf049027i]

Lv, H.P., Zhong, Q.S., Lin, Z., et al., 2012. Aroma characterisation of $\mathrm{Pu}$-erh tea using headspace-solid phase micro-extraction combined with GC/MS and GColfactometry. Food Chem., 130(4):1074-1081. [doi:10. 1016/j.foodchem.2011.07.135]

Lyons, R., Manners, J.M., Kazan, K., 2013. Jasmonate biosynthesis and signaling in monocots: a comparative overview. Plant Cell Rep., 32(6):815-827. [doi:10.1007/ s00299-013-1400-y]

Martin, D.M., Gershenzon, J., Bohlmann, J., et al., 2003. Induction of volatile terpene biosynthesis and diurnal emission by methyl jasmonate in foliage of Norway spruce. Plant Physiol., 132(3):1586-1599. [doi:10.1104/ pp.103.021196]

Muthumani, T., Verma, D.P., Venkatesan, S., et al., 2013. Influence of climatic seasons on quality of south Indian black teas. J. Nat. Prod. Plant Resource, 3(1):30-39.

Ogawa, K., Ijima, Y., Guo, W., et al., 1997. Purification of a 
$\beta$-primeverosidase concerned with alcoholic aroma formation in tea leaves (cv. Shuixian) to be processed to oolong tea. J. Agric. Food Chem., 45(3):877-882. [doi:10. 1021/jf9605431]

Qin, Z., Pang, X., Chen, D., et al., 2013. Evaluation of Chinese tea by the electronic nose and gas chromatography-mass spectrometry: correlation with sensory properties and classification according to grade level. Food Res. Int., 53(2):864-874. [doi:10.1016/j.foodres.2013.02.005]

Qiu, D., Pan, X., Wilson, I.W., et al., 2009. High throughput sequencing technology reveals that the taxoid elicitor methyl jasmonate regulates microRNA expression in Chinese yew (Taxus chinensis). Gene, 436(1-2):37-44. [doi:10.1016/j.gene.2009.01.006]

Ravichandran, R., 2002. Carotenoid composition, distribution and degradation to flavour volatiles during black tea manufacture and the effect of carotenoid supplementation on tea quality and aroma. Food Chem., 78(1):23-28. [doi:10.1016/S0308-8146(01)00303-X]

Rawat, R., Gulati, A., Kiran Babu, G.D., et al., 2007. Characterization of volatile components of Kangra orthodox black tea by gas chromatography-mass spectrometry. Food Chem., 105(1):229-235. [doi:10.1016/j.foodchem. 2007.03.071]

Rodriguez-Saona, C., Crafts-Brandner, S.J., ParÉ, P.W., et al., 2001. Exogenous methyl jasmonate induces volatile emissions in cotton plants. J. Chem. Ecol., 27(4):679-695. [doi:10.1023/A:1010393700918]

Sanderson, G.W., Co, H., Grahamm, H.N., 1973. Formation of black tea aroma. J. Agric. Food Chem., 21(4):576-585. [doi:10.1111/j.1365-2621.1971.tb04031.x]

Sarry, J.E., Günata, Z., 2004. Plant and microbial glycoside hydrolases: volatile release from glycosidic aroma precursors. Food Chem., 87(4):509-521. [doi:10.1016/j. foodchem.2004.01.003]

Schuh, C., Schieberle, P., 2006. Characterization of the key aroma compounds in the beverage prepared from Darjeeling black tea: quantitative differences between tea leaves and infusion. J. Agric. Food Chem., 54(3):916-924. [doi:10.1021/jf052495n]

Tomlins, K.I., Mashingaidze, A., 1997. Influence of withering, including leaf handling, on the manufacturing and quality of black teas-a review. Food Chem., 60(4):573-580. [doi:10.1016/S0308-8146(97)00035-6]

Vitzthum, O.G., Werkhoff, P., Hubert, P., 1975. New volatile constituents of black tea aroma. J. Agric. Food Chem., 23(5):999-1003. [doi:10.1021/jf60201a032]

Wakuta, S., Hamada, S., Ito, H., et al., 2010. Identification of a $\beta$-glucosidase hydrolyzing tuberonic acid glucoside in rice (Oryza sativa L.). Phytochemistry, 71(11-12): 1280-1288. [doi:10.1016/j.phytochem.2010.04.025]

Wang, D., Kurasawa, E., Yamaguchi, Y., et al., 2001. Analysis of glycosidically bound aroma precursors in tea leaves. 2 . Changes in glycoside contents and glycosidase activities in tea leaves during the black tea manufacturing process. $J$. Agric. Food Chem., 49(4):1900-1903. [doi:10.1021/ jf001077+]

Yamanishi, T., Kobayashi, A., Nakamura, H., et al., 1968a. Flavor of black tea. Agric. Biol. Chem., 32(3):379-386.

Yamanishi, T., Wickremasinghe, R.L., Perera, K.P.W.C., 1968b. Studies on the quality and flavour of tea. Part 3. Gas chromatographic analyses of the aroma complex. Tea Quart., 39:81-86.

Yang, Z., Baldermann, S., Watanabe, N., 2013. Recent studies of the volatile compounds in tea. Food Res. Int., 53(2): 585-599. [doi:10.1016/j.foodres.2013.02.011]

\section{中文概要:}

\section{本文题目: 茉莉酸甲酯诱导茶鲜叶制成的红茶中香气成分变化}

\section{Aroma changes of black tea prepared from methyl jasmonate treated tea plants}

研究目的: 为茉莉酸甲酯诱导来提高茶叶香气品质提供理论依据, 为新型花香红茶的研制提供技术支持。

创新要点: 首次将茉莉酸甲酯应用于诱导茶叶香气品质提高, 初步验证了茶叶香气品质提高的本质原因: 相关酶活性提高，基因表达上调。

研究方法: 采用顶空固相微萃取法 (HS-SPME) 对红茶香气进行富集, 气相色谱-质谱联用仪 (GC-MS) 进行解吸附分析, 实时定量多聚酶链式反应（qRT-PCR）分析茶鲜叶中香气相关酶基因表达。

重要结论：茉莉酸甲酯诱导后的茶鲜叶中多酚氧化酶（PPO）活性上升， $\beta$-葡萄糖苷酶活性下降; PPO 和 $\beta$-樱草糖苷酶基因表达上调， $\beta$-葡萄糖苷酶基因表达下调。茉莉酸甲酯诱导后的茶鲜叶能明显 提高由其制成的红茶香气品质, 且萜烯醇类和萜烯类含量明显提高。

关键词组: 红茶; 香气; 茉莉酸甲酯; 顶空固相微萃取（HS-SPME） 